\title{
Large-Scale GPU Simulations of Granular Flows and Fluidized Beds: An LBM-DEM Coupling Approach
}

\author{
C. RADEKE $^{1}$, S. RADL ${ }^{2}$, R. SUngkoRn ${ }^{2}$, D. SuzZI ${ }^{1}$, J. G. KHINASt ${ }^{1,2}$ \\ ${ }^{1}$ Research Center Pharmaceutical Engineering (RCPE), Graz, Austria \\ 2 Institute for Process and Particle Engineering (IPPT), Graz University of Technology, Graz, Austria \\ E-mails: charles.radeke@rcpe.at (C. Radeke), khinast@tugraz.at (J. G. Khinast)
}

Sci Pharm. 2010; 78: 644

doi:10.3797/scipharm.cespt.8.PMS17

\begin{abstract}
Granular flows are extremely important for the pharmaceutical and chemical industry, as well as for other scientific branches dealing with granular media. Thus, the understanding of the impact of particle size and related effects on the mean as well as on the fluctuating flow field in granular flows is critical for design and optimization of powder processing.

We use a specialized simulation tool written in C and CUDA, a massive parallelization technique which runs on the Graphics Processing Unit (GPU). It was chosen to be able to simulate significantly higher amounts of particles in acceptable runtime.

We focus on flow fields and wet-mixing properties obtained in the lower million particle range. In our talk, we present simulation results of a pitched blade mixer on a laboratory scale. Particles with a medium particle size of 365 micrometers are blended at 30-60 rpm for several turns (> 10 revolutions). The GPU implementation enable the DEM simulation of quartz powders in the real geometric resolution. In order to investigate the mixing process during the continuous spraying of a liquid, the DEM code was extended suitable. Liquid films and bridges implement the spread of the fluid in the aggregates. Depending on the wetness level on particle surfaces, capillary forces account for cohesion and agglomeration. The simulations will shed light on the optimum location and the type of fluid entry, and allow predictions about the time until a homogeneous moisture distribution in a blended bulk can be achieved.

Complex process engineering like drying of fluidized particles in hot air streams is a huge challenge for numerical simulations. Highly resolved two-phase flow of one million particles will be investigated. Here we focus on both, a gas-phase simulated using the Lattice Boltzmann Method (LBM) and a coupled solid-phase of discrete particles, simulated by using Discrete Element method (DEM). Subgrid scale two-way coupling of liquid and solid flow allows the investigation of residence time as well as other important process parameters and the prediction of an optimum operation regime.
\end{abstract}

